\title{
A New Multiple Antennas Method based Energy Detector for Cognitive Radio over Fading Channels
}

\author{
Mahmood A. Abdulsattar \\ E. E. Department \\ College of Engineering \\ University of Baghdad \\ Baghdad, Iraq
}

\author{
Zahir A. Hussein \\ E. E. Department \\ College of Engineering \\ University of Baghdad \\ Baghdad, Iraq
}

\begin{abstract}
In this paper we presented a DSP processor to be used as a baseband energy detectorbased spectrum sensingfor cognitive radio (CR) under fading channels. We Derived expression for the average probability of detection over Rayleigh fading channels in easiest form and Nakagami- $m$ channels with no diversity. Novelties of this work came from proposing a multiple antennas signal detection technique for spectrum sensing in a multipath fading environment. Without involving the sampling theorems and the accompanying approximation, we derive closed-form expressions for the probability of detection and false alarm over an additive white Gaussian noise (AWGN) channels for the proposed method. The proposed technique is a low complexity scheme by using two energy detectors and not need prior information about the channel gain in comparison with other methods.
\end{abstract}

\section{Keywords}

Real-time Implementation, Cognitive Radio (CR), Energy Detection, Fading Channels, Square Law Combining.

\section{INTRODUCTION}

Wireless communication systems were growth significantly over the last two decades. However, there are limits to growth, because the radio spectrum used for wireless communications is a finite resource. A band assigned to a primary user may be absolutely free or idle at a particular time or geographical area. In [1], Spectrum utilization is a function of time and location; it ranges from $15 \%$ to $85 \%$ according to the federal communications commission (FCC).

As a solution for the spectrum used inefficiently problem, cognitive radio (CR) proposes an opportunistic spectrum usage approach [2], in which frequency bands that are not being used by their licensed users, also called the primary user (PU), are utilized by cognitive radios, also called the second user (SU).

In its most basic form, $\mathrm{CR}$ is a hybrid technology involving software defined radio (SDR). The basic concept of the SDR is that the radio can be totally configured or defined by the software like mixer, filters, modulators, demodulators [3], [4]. Thus, simply modifying the software, CR can completely change its functionality, or improve its performances, without replacing hardware. This characteristic allows adding new features to $\mathrm{CR}$ and improving its existing ability.

Currently, there are varieties of techniques which are commonly used in signal processing techniques to identify if a channel is occupied or vacant [5]: matched filter, cyclostationary feature detection and energy detection.

In [6], energy detection (a.k.a. non-coherent detection), is the signal detection mechanism using an energy detector (a.k.a. radiometer), works by measuring the received signal energy and deciding on the presence or absence of the PU, by comparing the received energy level with a threshold as shown in Fig. 1.

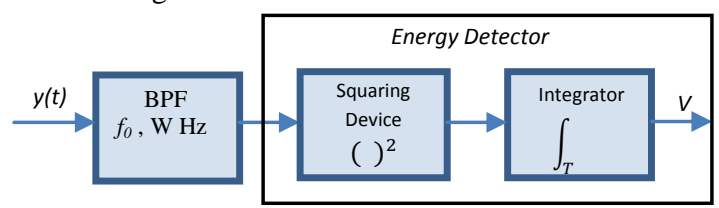

Fig 1: Classical model of energy detector

In low signal-to-noise ratio (SNR) the secondary user cannot sense primary user due to an obstacle or fade effect, therefore hidden primary user problem must be alleviated. In [7], to reduce the multipath and shadowing effects of the wireless channels, diversity reception (i.e. multiple antennas) techniques provide promising solutions as to improve BER performances. In [8] diversity reception has long been familiar as a great communication receiver technique for alleviating the harmful effects of channel fading and cochannel interference. Diversity means the providing of two or more independent fading paths from transmitter to receiver. These independent signals are combined in a special way to increase the overall SNR. Diverse methods can be categorized in accordance with the type of combining used at the receiver, namely, equal gain combining (EGC), maximal-ratio combining (MRC) and selection diversity combining (SDC).

In this paper we derive the average probability of detection over Rayleigh fading channels in simplest form. A new technique of signal detecting is proposed. The proposed scheme has minimum hardware used.

The paper is organized as follows. Section II lists the main issues of related works. Section III explains briefly the description of the baseband energy detector system model. Drives average probability of detection for baseband energy detector over Rayleigh fading Channels in section IV. Section $\mathrm{V}$ gives a description of the proposed method using modified square law combining (SLC) diversity technique and derives probability equations under AWGN channels. Finally, in section VI, the conclusions are mentioned.

\section{SYSTEM MODEL}

A common method for detection of unknown signals in noise is energy detection. The advantage of this technique is that it does not require knowledge of the transmitted signal by the $\mathrm{PU}$, but it has the disadvantage of requiring an accurate estimation of the received ambient noise power.

A system model of energy detector with baseband sampling for CR can be shown in Fig. 2. 


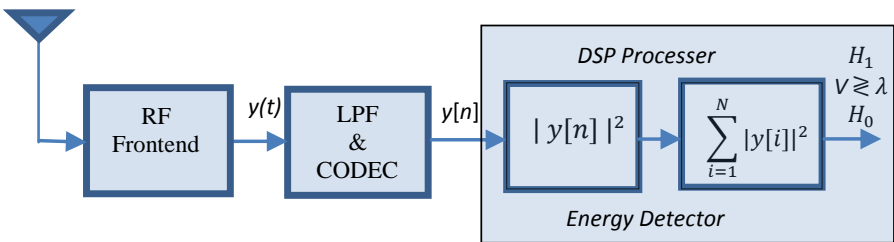

Fig 2: An energy detector with baseband sampling.

In order to measure the energy of the received signal, the output signal of CODEC is squared and integrated over the sensing interval $T_{S}$. The sensing interval $T_{S}$ it's usually assumed to be small enough so that the PU signal can span over the whole sensing interval. According to the Nyquist sampling theorem, the minimum sampling rate should be $F_{s a} \geq 2 W$, where $W$ is the highest frequency of the original signal, hence, the minimum sample size $N$ collected by the energy detector can be represented as $2 T W$. In realtime $2 W$ equal to sampling frequency $F_{s a}$ of the DSP card, hence as sensing time $T_{S}$ is chosen such that $N$ is an integer

$$
N=T_{s} \quad F_{s a} .
$$

In [9], in detection theory for $\mathrm{CR}$ systems we want to determine whether a PU is present or not. In a binary hypothesis test, the received signal after CODEC can be given as

$$
\left\{\begin{array}{l}
H_{0}: y[n]=w[n] \\
H_{1}: y[n]=h \cdot x[n]+w[n] \quad n=0, \ldots, N-1
\end{array}\right.
$$

where $H_{0}$ represents the hypothesis corresponding to "no signal transmitted", and $H_{1}$ to "signal transmitted", $x[n]$ is unknown deterministic transmitted signal, $w[n]$ is an additive white Gaussian noise (AWGN) with zero mean and variance $\sigma_{n}^{2}$, is known a priori, $N$ denotes the number of samples collected during the signal sensing period and $h$ amplitude of channel gain (channel coefficient).

The test statistic for an energy detector with predetermined threshold $\lambda$ is defined as follows

$$
V=\sum_{i=1}^{N}|y[i]|^{2} \underset{H_{0}}{\stackrel{H_{1}}{\gtrless} \lambda}
$$

The most often used approaches in the energy detection are based on the Neyman-Pearson (NP) lemma. The NP detection criterion enlarges the probability of detection $\left(P_{\mathrm{d}}\right)$ for a given probability of false alarm $\left(P_{\mathrm{fa}}\right)$.

It's easy to show that the $P_{\mathrm{fa}}$ under AWGN channels presented in [10], is the same for energy detector under baseband sampling

$$
P_{\mathrm{fa}}=\frac{\Gamma\left(k, \frac{\lambda}{2 \sigma_{n}^{2}}\right)}{\Gamma(k)}
$$

where $k=N / 2$, is an integer, $\Gamma($.$) is the gamma function,$ which is defined as $\Gamma(u)=\int_{0}^{\infty} t^{u-1} e^{-t} d t$, and incomplete gamma function is given by $\Gamma(a, s)=\int_{s}^{\infty} t^{a-1} e^{-t} d t$ [11].

Also the $P_{\mathrm{d}}$ under AWGN channels for baseband sampling can be expressed in term of the generalized Marcum

$Q-$ function as

$$
P_{\mathrm{d}}=Q_{k}\left(\sqrt{N \gamma}, \frac{\sqrt{\lambda}}{\sigma_{n}}\right)
$$

\section{ENERGY DETECTOR UNDER FADING CHANNELS WITH NO DIVERSITY}

In this section, we derive the average detection probability over Rayleigh and Nakagami- $m$ fading channels with asingle antenna, under $H_{1}$ hypothesis. $P_{\mathrm{fa}}$ in this case will remain the same, since it is independent of the presence of signal.

In [12], fading channels can be roughly divided into two types: (1) Large-scale fading due to (a) Path loss and (b) Shadowing, both fading is typically frequency independent, shadow fading represented by a log-normal distribution. (2) Small-scale fading due the time spreading of the signal and the time varying behavior of the channel. A Doppler shift causes the time varying behavior of the channel. The time spreading in a multipath environment causes the signal to undergo either flat or frequency selective fading. If the channel has a constant gain and linear phase response over a bandwidth that is greater than the bandwidth of the transmitted signal, then the received signal undergoes flat fading. It can be modeled as a Rayleigh, Rician, or Nakagami distribution.

In [8], in urban regions where the line-of-sight component is often blocked by obstacles, the Rayleigh, the process was suggested as a suitable stochastic model process. In rural regions, however, the line-of-sight component is often a part of the received signal, so that the Rice process is the more suitable stochastic model for these channels. In reality, signals in a wireless radio environment are affected by both lognormal and Rayleigh fading [13]

\subsection{Flat Fading}

A) Nakagami-m fading channels: We consider Nakagamimfading channels, which can model a variety of fading effects. The probability density function (PDF) of $\gamma$ over Nakagami- $m$ channel is [14]

$$
f(\gamma)=\frac{1}{\Gamma(m)}\left(\frac{m}{\bar{\gamma}}\right)^{m} \gamma^{m-1} \exp \left(-\frac{m \gamma}{\bar{\gamma}}\right), \quad \gamma \geq 0
$$

where $\gamma=\frac{h^{2} \sigma_{s}^{2}}{\sigma_{n}^{2}}$ is SNR, $\bar{\gamma}=\frac{\sigma_{s}^{2}}{\sigma_{n}^{2}}$ is the flat fading average SNR (averaging over $h^{2}$ ), $\sigma_{s}^{2}$ variance of signal, $m$ is the Nakagami parameter. Nakagami- $m$ follows Rayleigh fading when $m=1$, for $m=\frac{(k+1)^{2}}{(2 k+1)}$ the distribution is approximately Rician fading with parameter $k$, and for $m=\infty$ there is no fading. The average probability of detection (averaged over the fading) is

$$
\bar{P}_{\mathrm{d}, N a k}=\int_{0}^{\infty} f(\gamma) P_{\mathrm{d}}(\gamma) d \gamma
$$

The Marcum-Q function in (5), can be expressed as given in [15, eq. (4.74), pp. 104]

$$
Q_{k}\left(\sqrt{N \gamma}, \frac{\sqrt{\lambda}}{\sigma_{n}}\right)=\sum_{n=0}^{\infty} \frac{\left(\frac{N \gamma}{2}\right)^{n} e^{-\frac{N \gamma}{2}}}{n !} \sum_{u=0}^{n+k-1} \frac{\left(\frac{\lambda}{2 \sigma_{n}^{2}}\right)^{u} e^{-\frac{\lambda}{2 \sigma_{n}^{2}}}}{u !}
$$

we can write (7) as

$$
\begin{gathered}
\bar{P}_{\mathrm{d}, N a k}=\frac{e^{-\frac{\lambda}{2 \sigma_{n}^{2}}}}{\Gamma(m)}\left(\frac{m}{\bar{\gamma}}\right)^{m} \sum_{\substack{n=0 \\
n+1}}^{\infty} \frac{1}{n !}\left(\frac{N}{2}\right)^{n} \\
\int_{0}^{\infty} x^{n+m-1} e^{-\left(\frac{N}{2}+\frac{m}{\bar{\gamma}}\right) x} d x \times \sum_{u=0}^{n+k} \frac{1}{u !}\left(\frac{\lambda}{2 \sigma_{n}^{2}}\right)^{u}
\end{gathered}
$$


the integral in (9) can be evaluated as [11]

$$
\int_{0}^{\infty} x^{n+m-1} e^{-\left(\frac{N}{2}+\frac{m}{\bar{\gamma}}\right) x} d x=(n+m-1) !\left(\frac{N}{2}+\frac{m}{\bar{\gamma}}\right)^{-n-m}
$$

sub (10) in (9) we get

$$
\begin{aligned}
& \bar{P}_{\mathrm{d}, N a k}=\frac{e^{-\frac{\lambda}{2 \sigma_{n}^{2}}}}{\Gamma(m)}\left(\frac{m}{\bar{\gamma}}\right)^{m} \sum_{n=0}^{\infty} \frac{(n+m-1) !}{n !}\left(\frac{N}{2}\right)^{n} \\
& =\frac{\left(\frac{N}{2}+\frac{m}{\bar{\gamma}}\right)^{-n-m} \times \sum_{u=0}^{n+k-1} \frac{1}{u !}\left(\frac{\lambda}{2 \sigma_{n}^{2}}\right)^{u}}{\Gamma(m)}\left(\frac{2 m}{2 m+N \bar{\gamma}}\right)^{m} \sum_{n=0}^{\infty} \frac{(n+m-1) !}{n !}\left(\frac{N \bar{\gamma}}{2 m+N \bar{\gamma}}\right)^{n} \\
& \times \sum_{u=0}^{n+k-1} \frac{1}{u !}\left(\frac{\lambda}{2 \sigma_{n}^{2}}\right)^{u} .
\end{aligned}
$$

The result in (12) is an alternative derivation of average probability of detection over Nakagami-mchannel.

B) Rayleigh channels: The average probability over Rayleigh channel can be calculated when $m=1$ in (12), thus

$$
\begin{gathered}
\bar{P}_{\mathrm{d}, \text { Ray }}=\left(\frac{2}{2+N \bar{\gamma}}\right) e^{-\frac{\lambda}{2 \sigma_{n}^{2}}} \sum_{n=0}^{\infty}\left(\frac{N \bar{\gamma}}{2+N \bar{\gamma}}\right)^{n} \\
\times \sum_{u=0}^{n+k-1} \frac{1}{u !}\left(\frac{\lambda}{2 \sigma_{n}^{2}}\right)^{u}
\end{gathered}
$$

dividing inner geometric series into two series

$$
\begin{aligned}
\bar{P}_{\mathrm{d}, \text { Ray }}= & \left(\frac{2}{2+N \bar{\gamma}}\right) e^{-\frac{\lambda}{2 \sigma_{n}^{2}}} \sum_{n=0}^{\infty}\left(\frac{N \bar{\gamma}}{2+N \bar{\gamma}}\right)^{n} \\
& \times\left[\sum_{u=0}^{k-1} \frac{1}{u !}\left(\frac{\lambda}{2 \sigma_{n}^{2}}\right)^{u}+\sum_{u=k}^{n+k-1} \frac{1}{u !}\left(\frac{\lambda}{2 \sigma_{n}^{2}}\right)^{u}\right] .
\end{aligned}
$$

In [16,eq. (2.5), pp. 24], the incomplete gamma function can be expressed as

$$
\Gamma(n, x)=(n-1) ! e^{-x} \sum_{i=0}^{n-1} \frac{x^{i}}{i !}
$$

based on (15), $P_{\mathrm{fa}}$ can be evaluated as

$$
P_{\mathrm{fa}}=e^{-\frac{\lambda}{2 \sigma_{n}^{2}}} \sum_{u=0}^{k-1} \frac{1 !}{u !}\left(\frac{\lambda}{2 \sigma_{n}^{2}}\right)^{u} .
$$

the first part of (14) can be simplified as

$$
\begin{gathered}
\left(\frac{2}{2+N \bar{\gamma}}\right) \sum_{n=0}^{\infty}\left(\frac{N \bar{\gamma}}{2+N \bar{\gamma}}\right)^{n} e^{-\frac{\lambda}{2 \sigma_{n}^{2}}} \sum_{u=0}^{k-1} \frac{1}{u !}\left(\frac{\lambda}{2 \sigma_{n}^{2}}\right)^{u} \\
=\left(\frac{2}{2+N \bar{\gamma}}\right) \frac{e^{-\frac{\lambda}{2 \sigma_{n}^{2}}}}{1-\frac{N \bar{\gamma}}{2+N \bar{\gamma}}} \sum_{u=0}^{k-1} \frac{1}{u !}\left(\frac{\lambda}{2 \sigma_{n}^{2}}\right)^{u}=P_{\mathrm{fa}}
\end{gathered}
$$

sub in (14), we get

$$
\begin{aligned}
\bar{P}_{\mathrm{d}, \text { Ray }}=P_{\mathrm{fa}}+ & \left(\frac{2}{2+N \bar{\gamma}}\right) e^{-\frac{\lambda}{2 \sigma_{n}^{2}}} \sum_{n=0}^{\infty}\left(\frac{N \bar{\gamma}}{2+N \bar{\gamma}}\right)^{n} \\
& \times \sum_{u=k}^{n+k-1} \frac{1}{u !}\left(\frac{\lambda}{2 \sigma_{n}^{2}}\right)^{u}
\end{aligned}
$$

rearranging the index of the two series in as

$$
\begin{aligned}
\bar{P}_{\mathrm{d}, \text { Ray }}=P_{\mathrm{fa}} & +\left(\frac{2}{2+N \bar{\gamma}}\right) e^{-\frac{\lambda}{2 \sigma_{n}^{2}}} \sum_{u=k}^{\infty} \frac{1}{u !}\left(\frac{\lambda}{2 \sigma_{n}^{2}}\right)^{u} \\
\times & \sum_{n=u-k+1}^{\infty}\left(\frac{N \bar{\gamma}}{2+N \bar{\gamma}}\right)^{n}
\end{aligned}
$$

evaluating the inner geometric series will be

$$
\begin{aligned}
\sum_{n=u-k+1}^{\infty}\left(\frac{N \bar{\gamma}}{2+N \bar{\gamma}}\right)^{n} & \\
& =\left(\frac{N \bar{\gamma}}{2+N \bar{\gamma}}\right)^{u}\left(\frac{2+N \bar{\gamma}}{N \bar{\gamma}}\right)^{k-1}(2 \\
& +N \bar{\gamma})
\end{aligned}
$$

sub in (19) we get

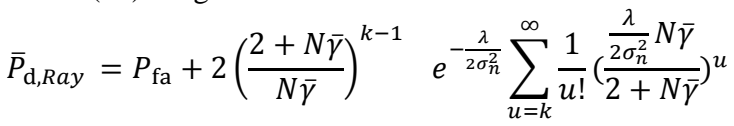

the remaining infinite series changed by using exponential function

$$
\begin{aligned}
& \sum_{u=k}^{\infty} \frac{1}{u !}\left(\frac{\frac{\lambda}{2 \sigma_{n}^{2}} N \bar{\gamma}}{2+N \bar{\gamma}}\right)^{u}=\sum_{u=0}^{\infty} \frac{1}{u !}\left(\frac{\frac{\lambda}{2 \sigma_{n}^{2}} N \bar{\gamma}}{2+N \bar{\gamma}}\right)^{u} \\
& -\sum_{u=0}^{k-1} \frac{1}{u !}\left(\frac{\frac{\lambda}{2 \sigma_{n}^{2}} N \bar{\gamma}}{2+N \bar{\gamma}}\right)^{u}=e^{\frac{\lambda}{2 \sigma_{n}^{2} N \bar{\gamma}}}-\sum_{u=0}^{k-1} \frac{1}{u !}\left(\frac{\frac{\lambda}{2 \sigma_{n}^{2}} N \bar{\gamma}}{2+N \bar{\gamma}}\right)^{u}
\end{aligned}
$$

sub (22) in (21), simplify, using (15), we get

$\bar{P}_{\mathrm{d}, \text { Ray }}=P_{\mathrm{fa}}+2\left(\frac{2+N \bar{\gamma}}{N \bar{\gamma}}\right)^{k-1} e^{-\frac{\frac{\lambda}{\sigma_{n}^{2}}}{2+N \bar{\gamma}}}\left[1-\frac{\Gamma\left(\mathrm{k}, \frac{\frac{\lambda}{2 \sigma_{n}^{2}} N \bar{\gamma}}{2+N \bar{\gamma}}\right)}{\Gamma(k)}\right]$

This closed-form expression for the average probability of detection over Rayleigh channels in a simpler form than in [14], [17] and [18].

\subsection{Shadow Fading}

In [19], the most common model for shadow fading is the lognormal distribution. This model has been confirmed empirically to accurately model the variation in receiving power in both outdoor and indoor radio propagation environments. The log-normal distribution is represented by the following equation:

$$
f\left(x_{d B}\right)=\frac{1}{\sqrt{2 \pi} \sigma_{d B}} \exp \left(-\frac{\left(x_{d B}-\mu_{d B}\right)^{2}}{2 \sigma_{d B}^{2}}\right) .
$$

When $\gamma$ is log-normal distributed, the authors proposed that the probability of detection can be evaluated numerically. 


\section{MODIFIED SQUARE LAW COMBINING (MSLC) METHOD}

We have studied some diversities techniques that have "one energy detector" such as selection combining (SC) [14], [20] maximum ratio combining (MRC) [20], and equal gain combining (EGC) [14] we found it required complete knowledge of channel gain. For methods required "an energy detector for each antenna" such as a square law combining (SLC) and square law selection (SLS) [17], it's evident that they required energy detectors equal to the number of antennas which of course raise the implementationcosts. Taking into consideration the drawback of the methods proposed in [14], [17], [20] we investigate a method which required only one two energy detector and does not require any previous knowledge on the wireless channel gain.

In this diversity method the first energy detector sums the output of squared signals come from multiple antennas, and the second energy detector summing the statistics comes from the first energy detector and compared with a threshold to make a decision, as shown in Fig. 3.

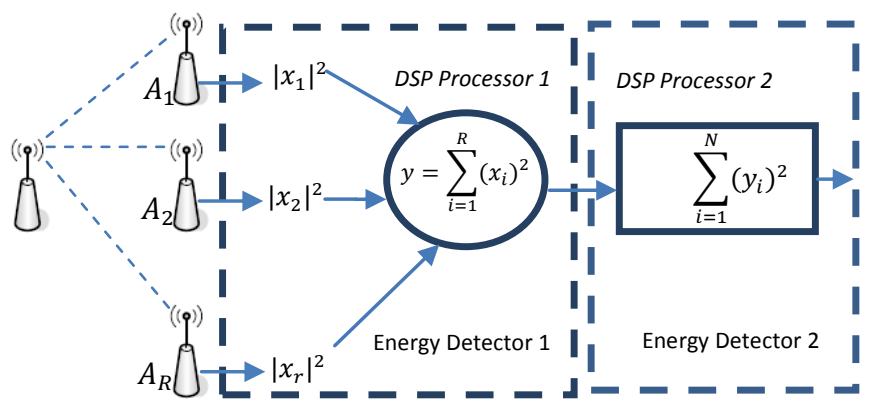

Fig. 3: Modified square law combining (MSLC) scheme

The equation for MSLC can be derived as follows

$$
\begin{aligned}
& y=\sum_{i=1}^{R}\left(x_{i}\right)^{2} \\
& V=\sum_{i=1}^{N}\left(y_{i}\right)^{2}
\end{aligned}
$$

if we assume the number of antennas used is $R$ and the symbol length is assumed to be $N$ then we get $N R$ Gaussian noise with zero mean and variance $\sigma_{n}^{2}$ at the output to compare with a threshold

$$
V_{\mathrm{MSLC}}=\sum_{i=1}^{N R}\left(y_{i}\right)^{2} \text {. }
$$

Since $V_{\mathrm{MSLC}}$ is a square sum of $N R A W G N$ with zero mean, i.e. $w[n] \sim \mathcal{N}\left(0, \sigma_{n}^{2}\right)$, thus the distribution of the test statistic is a chi-square with $N R$ degrees of freedom (DOF) $V_{M S L C} \sim \chi_{N R}^{2}$, can be evaluated as follows

$$
f_{V \mid H_{0}}(v)=\frac{1}{\sigma_{n}^{N R} 2^{\beta} \Gamma(\beta)} v^{\beta-1} e^{-v / 2 \sigma_{n}^{2}}, \quad v>0
$$

where $\beta=N R / 2$, is an integer. From the definition of false alarm probability, the $\mathrm{CR}$ decides in favor of $H_{1}$ while the band is idle, thus, the false alarm probability can be expressed as

$$
\begin{aligned}
P_{\mathrm{fa}(\mathrm{MSLC})} & =\operatorname{Pr}\left\{V_{\mathrm{MSLC}}>\lambda / H_{0}\right\}=\int_{v_{1}=\lambda}^{+\infty} f_{V \mid H_{0}}(v) d v \\
& =\frac{1}{\sigma_{n}^{N R} 2^{\beta} \Gamma(\beta)} \int_{\lambda}^{\infty} v^{\beta-1} e^{-v / 2 \sigma_{n}^{2}} d v
\end{aligned}
$$

to solve this equation, we must apply some variable conversion for $t=\frac{v}{2 \sigma_{n}^{2}}$, we get

$$
P_{\mathrm{fa}(\mathrm{MSLC})}=\frac{1}{\Gamma(\beta)} \int_{\frac{\lambda}{2 \sigma_{n}^{2}}}^{\infty} t^{\beta-1} e^{-t} d t
$$

by using the incomplete gamma function, therefore (30) becomes

$$
P_{\mathrm{fa}(\mathrm{MSLC})}=\frac{\Gamma\left(\beta, \frac{\lambda}{2 \sigma_{n}^{2}}\right)}{\Gamma(\beta)}
$$

using this formula, we can find a threshold to set achieved various false alarm probabilities for MSLC.

The same approach is applied when the signal of PU, $x[n]$ is present hence, the test statistic under hypothesis $H_{1}$ becomes

$$
V_{\mathrm{MSLC}}=\sum_{i=1}^{N R}\left(\begin{array}{lll}
x & {[i]+w} & {[i]}
\end{array}\right)^{2} .
$$

We can observe that $V_{\text {MSLC }}$ consists of two terms: a fixed (non-random) component $x[n]$ and a noise component $w[n]$ obey the Gaussian distribution. More specifically, $V$ is a noncentral chi-square distribution with non-central parameter $\sum_{i=1}^{N R} x^{2}[i]=N R \sigma_{s}^{2} \quad$ and $\quad N R \quad \mathrm{DOF}, V \sim \chi_{N R}^{2}\left(N R \sigma_{s}^{2}\right), \quad$ in particular, the PDF of $V_{\text {MSLC }}$ under $H_{1}$ hypothesis takes the form

$$
f_{V \mid H_{1}}(v)=\frac{1}{2 \sigma_{n}^{2}}\left(\frac{v}{N R \sigma_{s}^{2}}\right)^{\frac{\beta-1}{2}} e^{-\frac{N R \sigma_{s}^{2}+v}{2 \sigma_{n}^{2}}} I_{\beta-1}\left(\frac{\sqrt{v N R \sigma_{s}^{2}}}{\sigma_{n}^{2}}\right),
$$

where $I_{n}$ is the $n$ order modified Bessel function. The probability of detection is

$$
\begin{aligned}
& P_{\mathrm{d}(\mathrm{MSLC})}=\operatorname{Pr}\left\{V_{M S L C}>\lambda / H_{1}\right\}=\int_{v_{1}=\lambda}^{+\infty} f_{V \mid H_{1}}(v) d v \\
& =\int_{\lambda}^{+\infty} \frac{1}{2 \sigma_{n}^{2}}\left(\frac{v}{N R \sigma_{S}^{2}}\right)^{\frac{\beta-1}{2}} e^{-\frac{N R \sigma_{S}^{2}+v}{2 \sigma_{n}^{2}}} I_{\beta-1}\left(\frac{\sqrt{v N R \sigma_{s}^{2}}}{\sigma_{n}^{2}}\right) d v .
\end{aligned}
$$

The $P_{\mathrm{d}(\mathrm{MSLC})}$ can be expressed in term of the generalized Marcum $Q$-function, which is defined as [10]

$Q_{m}(\alpha, \eta)=\int_{\eta}^{\infty} x\left(\frac{x}{\alpha}\right)^{m-1} \exp \left(-\frac{x^{2}+\alpha^{2}}{2}\right) I_{m-1}(\alpha x) d x$ 
where $m$ is a nonnegative integer, and $\alpha$ and $\eta$ are nonnegative real numbers, if we change variable of integration (36), $v$ to $x$, where $x=\frac{v}{\sigma_{n}^{2}}$, and let $\alpha^{2}=\frac{N R \sigma_{s}^{2}}{\sigma_{n}^{2}}$, we obtain

$$
P_{\mathrm{d}(\mathrm{MSLC})}=Q_{\beta}\left(\sqrt{N R \gamma}, \frac{\sqrt{\lambda}}{\sigma_{n}}\right) \text {. }
$$

If $\beta \geq 100$, i.e. $N R \geq 200$, the Marcum $Q$-function is difficult to calculate or to take its inverse, thus, we can use the central limit theorem (CLT), for the large number of sample, we can use the Gaussian distribution to approximation the chisquare distribution, under $H_{0}$ hypothesis, and non-central chisquare distribution, under $H_{1}$ hypothesis, thus, the CLT can therefore be employed to approximate the test statistic as Gaussian

$V_{M S L C} \sim\left\{\begin{array}{c}H_{0}: \mathcal{N}\left(N R \sigma_{n}^{2}, 2 N R \sigma_{n}^{4}\right) \\ H_{1}: \mathcal{N}\left(N R \sigma_{n}^{2}(1+\gamma), 2 N R \sigma_{n}^{4}(1+2 \gamma)\right) .\end{array}\right.$

If only AWGN is considered, $P_{\mathrm{fa}(\mathrm{MSLC})}$ and $P_{\mathrm{d}(\mathrm{MSLC})}$ of energy detector can be derived in terms of the $Q$ function as follows

$$
\begin{gathered}
P_{\mathrm{fa}(\mathrm{MSLC})}=Q\left(\frac{\lambda-N R \sigma_{n}^{2}}{\sqrt{2 N R} \sigma_{n}^{2}}\right) \\
P_{\mathrm{d}(\mathrm{MSLC})}=Q\left(\frac{\lambda-N R \sigma_{n}^{2}(1+\gamma)}{\sigma_{n}^{2} \sqrt{2 N R(1+2 \gamma)}}\right)
\end{gathered}
$$

where the $Q(x)=\frac{1}{2 \pi} \int_{x}^{\infty} \exp \left(-\frac{t^{2}}{2}\right) d t$ is standard $Q$-function [11]. The decision threshold $\lambda$ is determined by the pdf of the noise only signal, thus, by using (46), we get

$$
\lambda_{(\mathrm{MSLC})}=\left(\sqrt{2 N R} Q^{-1}\left(P_{\mathrm{fa}(\mathrm{MSLC})}\right)+N R\right) \sigma_{n}^{2}
$$

where $Q^{-1}($.$) denote the inverse Q$-function [16]. Thus, the threshold does not depend on the channel gain.

\section{NUMERICAL RESULTS}

We explain the effectiveness of the MSLC multiple antenna processingtechniques through the probability of detection for a pre-assumedprobability of falsealarm, $P_{\mathrm{fa}}=$ 0.01 , withdifferent SNRs varied from -5 to $15 \mathrm{~dB}$.We considered afourantenna system $(\mathrm{R}=4), N=10$, number of samples collected during the signal sensing.

In the Fig. $4, P_{\mathrm{d}}$ vs.SNR is illustrated.It is seen EGC and MLSC give the best implementation, when we consider EGC which required perfect channel gain knowledge.It's easy to say that MLSC technique is more suitable to be implemented and need only two energy detectors. As seen in figure SC and SLS are very close detection performance.

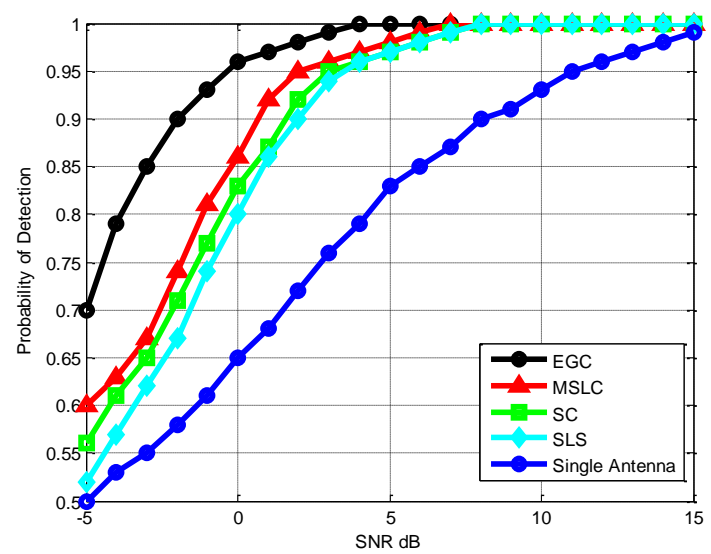

Fig. 4: $P_{d}$ vs.SNR

\section{CONCLUSION}

In this paper we present the idea was based on using the DSP processor card as energy detector. We derived closed form expressions for the average probability of detection in Rayleigh channels in simple form than in [12]-[14]. We proposed a modified method for spectrum sensing under fading channels. The advantage of this technique is using two energy detector and not need prior information about channels. In the present work, without involving the sampling theorems and the accompanying approximation, we derive the expressions for Probability of detection and false alarm over AWGN. Using this technique reduces the cost of the physical layer resources of CR. The enhancement in detection succeeded through the MLSC multiple antenna technique in energy detection is obvious.

\section{REFERENCES}

[1] FCC, "Spectrum policy task force report," in Proceedings of the Federal Communications Commission (FCC 2002), Washington, DC, USA, November 2002.

[2] J. Mitola and G. Q. Maguire, "Cognitive radio: making software radios more personal," IEEE Personal Communications, vol. 6, no. 4, pp. 13-18, 1999.

[3] J. Mitola, "The software radio architecture," IEEE Communications Magazine, pp. 26-38, May 1995.

[4] J. Mitola III, "Software radio architecture: a mathematical perspective," IEEE Journal on Selected Areas in Communication. vol. 17, no. 4, April 1999.

[5] J. Ma, G. Li, and B. H. Juang, "Signal processing in cognitive radio," Proceedings of the IEEE, vol. 97, no. 5, pp. $805-823$, may 2009.

[6] H. Urkowitz, "Energy detection of unknown deterministic signals," Proc. IEEE, vol. 55, no. 4, pp. 523-531, Apr. 1967.

[7] Haykin S. and M. Moher eds., Modern Wireless Communications. Prentice-Hall, 2004.

[8] M. Pätzold, Mobile Fading Channel., Article book, John Wiley \& Sons, Ltd, 2002. 
[9] Y. H. Zeng, Y. C. Liang, A. T. Hoang, and R. Zhang, "Review on Spectrum Sensing for Cognitive Radio: Challenges and Solutions," EURASIP Journal on Advances in Signal Processing, Volume 2010, pp.1-15.

[10] J. G. Proakis, Digital communications. fourth ed., McGraw-Hill, 2001.

[11] I. S. Gradshteyn and I. M. Ryzhik, Table of Integrals, Series, and Products. 7th ed. San Diego, CA: Academic, 2007.

[12] D. Tse and P. Viswanath, "Fundamentals of Wireless communication", Article book,Cambridge University Press, 2005.

[13] J. Lai and N. B. Mandayam, "Fade Margins for Minimum Duration Outages in Lognormal Shadow Fading and Rayleigh Fading", Proceedings of Asilomar, pp. 609-613, Monterrey, CA, November 1997.

[14] F. F. Digham, M. S. Alouini, and M. K. Simon, "On the energy detection of unknown signals over fading channels," Commun., vol. 5, pp. 3575-3579, May. 2003.

[15] M. K. Simon and M-S Alouini, Digital Communication over Fading Channels, New York: Wiley, 2 edition, 2005 .
[16] J. J. Lehtomaki, "The Detection and Correlation Modeling of Rayleigh Distribution Radar Signal," A Master Dissertation, Air Force Institute of Technology, University of Air, 1992. [Online] www.dtic.mil/cgibin/GetTRDoc?AD=ada256612.

[17] Digham,F., M. Alouini, and M. Simon, "On the Energy Detection of Unknown Signals Over Fading Channels," IEEE Transactions on Communications, vol. 55, pp. 2124, 2007.

[18] V. I. Kostylev, "Energy detection of a signal with random amplitude," in Proc. ICC 2002, New York, pp. 1606-1610,May 2002.

[19] D. Tse and P. Viswanath, "Fundamentals of Wireless communication", Article book,Cambridge University Press, 2005

[20] Ashish, P. and J. P. Linnartz, "Performance analysis of primary user detection in a multiple antenna cognitive radio," IEEE International Conference on Communications, vol. 7, pp. 6482-6486, 2007. 